\section{International relationships worth pursuing}

In a little more than one year, Darcy Marciniuk, an active Canadian 1 Thoracic Society (CTS) member and former CTS President (2006 to 2007), will make history and become president of the American College of Chest Physicians (ACCP) - one of the largest respiratory health organizations in the world. When I contemplate this fact, I cannot help but reflect on the value of CTS' relationships with the international community. Those relationships have effectively helped to set the stage for Darcy - a Canadian expert - to step into an important role in a neighboring country, and for the CTS to participate in bilateral and international projects that enhance respiratory health.

It would seem that from our earliest beginnings, the CTS viewed itself as being part of an international community. Our first medical director, Bill Jeanes, was recruited from Great Britain. Later, one of the Christmas Seal Visiting Professorships was awarded to a 'foreigner', Dr Jacques Chrétien, a department head in a Parisian (France) hospital. We obviously did not see ourselves then as being restricted to national boundaries.

Over the years, we have nourished relationships with organizations abroad and, in particular, with American and European organizations. We held joint scientific meetings with the American Thoracic Society (ATS) as early as 1975 and, as of 2001, we held our annual scientific meeting in conjunction with the ACCP CHEST Conference. CTS members have been regents-at-large on the ACCP Board of Regents, and have contributed as experts, officially and unofficially, to numerous clinical and research projects led by American and European organizations. Darcy Marciniuk co-chaired the 2005 CHEST Conference, which was held in Montreal, Quebec. Robert Levy, another former CTS President (2007 to 2008), co-chaired the 2010 CHEST Conference, which was held in Vancouver, British Columbia, and is the CTS' current representative to the European Respiratory Society (ERS). Other members have participated in the development of American cough (Louis-Philippe Boulet) and occupational asthma guidelines (Susan Tarlo, Jeremy Beach and Catherine Lemière), to name but a few of the international projects to which CTS members have contributed.

Today, Louis-Philippe Boulet is our official representative on the Guidelines International Network and the Global Alliance against Respiratory Diseases. His participation in these organizations enabled us to glean the best methodologies in the world for the development of clinical practice guidelines to apply those methodologies here at home, within the Canadian Respiratory Guidelines Committee. This has contributed to the CTS becoming an international leader in clinical practice guideline development.

The CTS has also been enriched through the involvement of members who are providing leadership to international projects of their own accord. For example, the involvement of Louis-Philippe Boulet, Mark FitzGerald and Paul O'Byrne as executive committee members of the Global Initiative for Asthma has informed the development of CTS asthma guidelines. Moreover, there are significant international research projects that include CTS members who are experts in their field (eg, Jean Bourbeau) and are also contributing to the CTS through the development of our own research strategy.

\section{Des relations internationales qui en valent la peine}

$\mathrm{D}$

ans un peu plus d'un an, Darcy Marciniuk, membre actif et ancien président de la SCT (2006-07), passera à l'histoire en devenant le président de l'American College of Chest Physicians (ACCP), l'une des plus importantes organisations au monde du domaine de la santé respiratoire. Lorsque je contemple ce fait, je ne puis m'empêcher de réfléchir à l'importance des relations qu'entretient la SCT avec la communauté internationale. Ces relations ont effectivement préparé la voie pour que Darcy, un expert canadien, assume un rôle important dans un pays voisin et pour que la SCT participe à des projets bilatéraux et internationaux en santé respiratoire.

Il semble que depuis ses tout débuts, la SCT se perçoit comme partenaire d'une communauté internationale. Notre premier directeur médical, Bill Jeanes, avait été recruté en Grande-Bretagne. Ultérieurement, une des chaires de professeur invité des Timbres de Noël fut décernée à un « étranger ", le docteur Jacques Chrétien, chef de département dans un hôpital parisien. Évidemment, à l'époque, nous ne nous percevions pas comme restreints par nos frontières nationales.

Au fil des ans, nous avons cultivé nos relations avec des organisations à l'étranger, et tout particulièrement avec des organisations américaines et européennes. Nous avons tenu des réunions scientifiques conjointement avec l'American Thoracic Society (ATS) dès 1975, et depuis 2001, nous tenons nos réunions scientifiques annuelles conjointement avec le congrès CHEST de l'ACCP. Des membres de la SCT ont siégé au conseil d'administration de l'ACCP à titre de membres hors cadre et ont contribué, officiellement et officieusement, à de nombreux projets dirigés par des organisations américaines et européennes. Darcy Marciniuk coprésida le congrès CHEST 2005 qui a eu lieu à Montréal. Robert Levy, un autre ancien président de la SCT (200708), coprésida le congrès CHEST 2010, qui a eu lieu à Vancouver, et est actuellement le représentant de la SCT auprès de la European Respiratory Society (ERS). D'autres membres ont participé à l'élaboration de lignes directrices américaines en matière de toux (Louis-Philippe Boulet) et d'asthme professionnel (Susan Tarlo, Jeremy Beach et Catherine Lemière), pour ne nommer que quelques-uns des projets internationaux auxquels des membres de la SCT ont contribué.

Actuellement, Louis-Philippe Boulet est notre représentant officiel auprès du Réseau pour les directives internationales et de l'Alliance Mondiale contre les Affections Respiratoires Chroniques. Sa participation à ces organisations nous a permis de glaner les meilleures méthodologies au monde en matière de lignes directrices de pratique clinique afin d'appliquer ces dernières chez nous, au sein du Comité des Lignes directrices canadiennes en santé respiratoire. Cela nous a aidés à devenir un chef de file international dans l'élaboration des lignes directrices de pratique clinique.

La SCT a aussi été enrichie par l'implication de membres qui, de leur propre chef, offrent un leadership à des projets internationaux. Par exemple, la participation de Louis-Philippe Boulet, Mark FitzGerald et Paul O'Byrne au comité exécutif du l'Initiative mondiale pour l'asthme a influencé l'élaboration de nos lignes directrices sur l'asthme. De plus, d'importants projets de recherche internationaux comprennent des membres de la SCT qui sont des experts dans 
We have been strengthening our relationships with our international partners through various means such as the following:

- Holding meetings with ACCP, ATS and ERS leaders to identify areas for collaboration;

- Presenting the CTS Scientific Program in conjunction with the ACCP CHEST Conference;

- Delivering, as part of the Canadian Respiratory Conference, ATS and joint ACCP/CTS sessions in which leading American experts deliver lectures on cutting-edge research;

- Establishing an annual CTS poster competition in conjunction with the ATS International Conference;

- Working on joint initiatives, such as the CTS/ERS fellowship exchange program, that will be launched this fall;

- Exploring the potential for the development of joint guidelines and continuing professional development programs; and

- Promoting CTS educational events and those of our partners to our respective members.

I am very grateful to our international partners for their collaboration. ACCP's generosity in hosting the CTS Scientific Program within its conference, and its facilitation of numerous other CTS activities held in conjunction with CHEST is particularly notable. Thank you ACCP, ATS and ERS, and thank you to all CTS members and staff who help to build and maintain these vital relationships.

We all know that it is a small world, and that science has no boundaries. Respiratory health can only benefit from our collaboration on the international stage.

It is my firm intention to continue to nourish these rich and rewarding relationships.

(Please note: CTS members involved abroad are numerous. If you are not mentioned in this article, but should be, please count yourself in and accept my apologies and sincere gratitude.)

Respectfully submitted, George A Fox MD MSc FRCPC FCCP President, Canadian Thoracic Society leur domaine (p. ex. Jean Bourbeau) et contribuent aussi à la SCT en participant à l'élaboration de notre propre stratégie en matière de recherche.

Nous avons renforcé les relations avec nos partenaires internationaux par divers moyens, notamment :

- La tenue de réunions avec les leaders de l'ACCP, l'ATS et l'ERS afin d'identifier des occasions de collaboration;

- La présentation du Programme scientifique de la SCT dans le cadre du congrès CHEST de l'ACCP;

- La présentation, dans le cadre du Congrès canadien sur la santé respiratoire, d'une séance de l'ATS et d'une séance conjointe de l'ACCP et la SCT, séances au cours desquelles des experts américains prononcent des discours sur la recherche de pointe;

- La création du Concours annuel d'affiches de la SCT dans le cadre du congrès international de l'ATS ;

- La collaboration sur des initiatives conjointes telles que le Programme d'échange de bourse de recherche avec ERS qui sera lancé cet automne ;

- L'étude sur la possibilité d'élaborer conjointement des lignes directrices et des programmes de développement professionnel ; et

- La promotion des activités de formation de la SCT et de celles de nos partenaires auprès de nos membres respectifs.

Je suis très reconnaissant de la collaboration de nos partenaires internationaux. La générosité dont fait preuve l'ACCP en accueillant le programme scientifique de la SCT au sein de son congrès et en permettant la tenue de nombre d'autres activités de la SCT conjointement avec ce congrès est particulièrement importante. J'offre mes remerciements à l'ACCP, l'ATS, l'ERS et à tous les membres de la SCT et du personnel qui aident à établir et à préserver ces relations importantes.

Nous savons tous que le monde est petit et que la science ne connaît pas de frontières; la santé respiratoire ne peut que tirer profit de notre collaboration sur la scène internationale.

J'ai fermement l'intention de continuer à nourrir ces relations enrichissantes!

(Veuillez noter : Les membres de la SCT impliqués à l'étranger sont nombreux. Si votre nom n'apparaît pas ci-haut tel qu'il l'aurait dû, comptez-vous tout de même parmi nos collaborateurs et recevez mes excuses ainsi que mes remerciements sincères.)

Salutations distinguées, George A Fox MD, M.Sc., FRCPC, FCCP Président, Société canadienne de thoracologie 


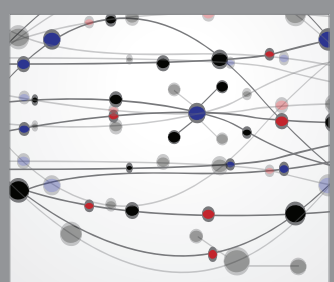

The Scientific World Journal
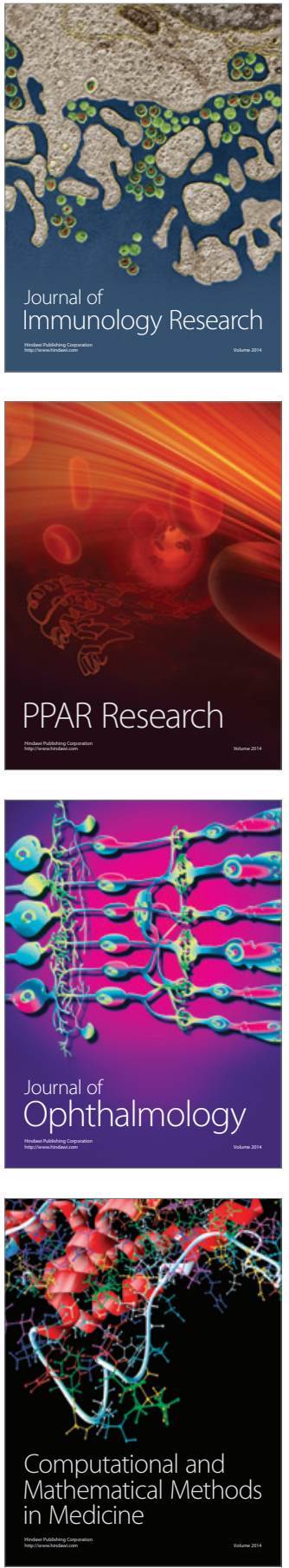

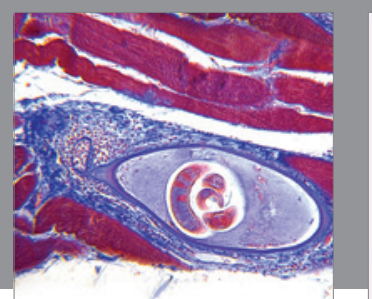

Gastroenterology Research and Practice

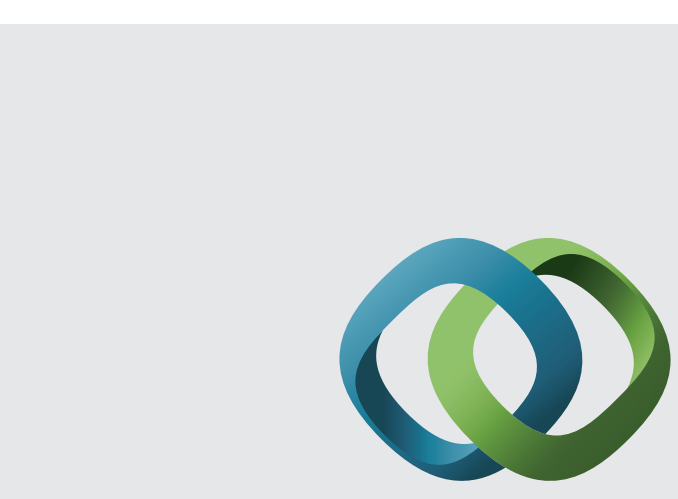

\section{Hindawi}

Submit your manuscripts at

http://www.hindawi.com
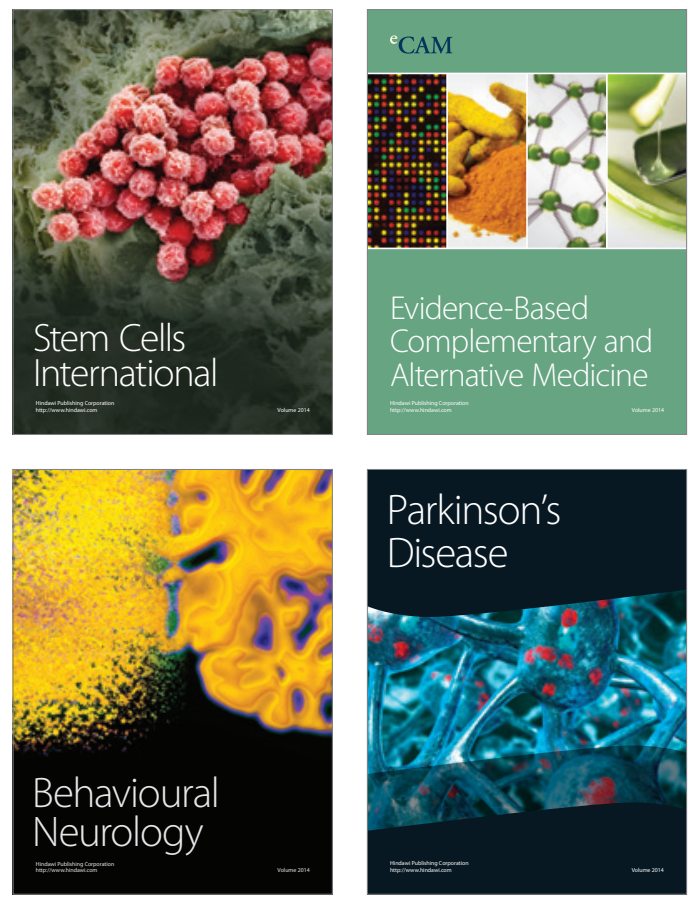
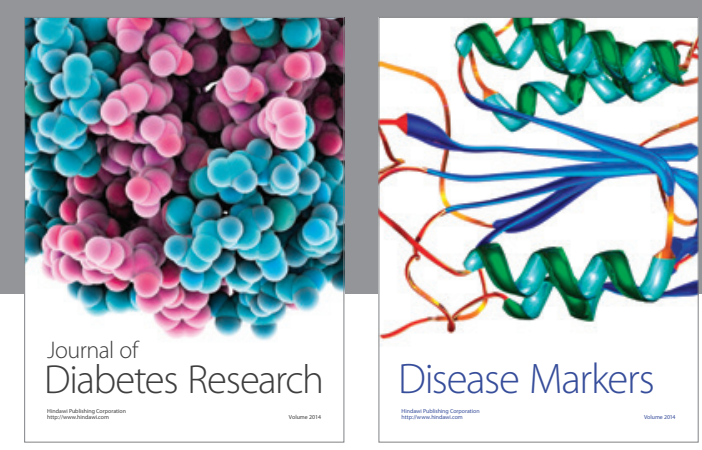

Disease Markers
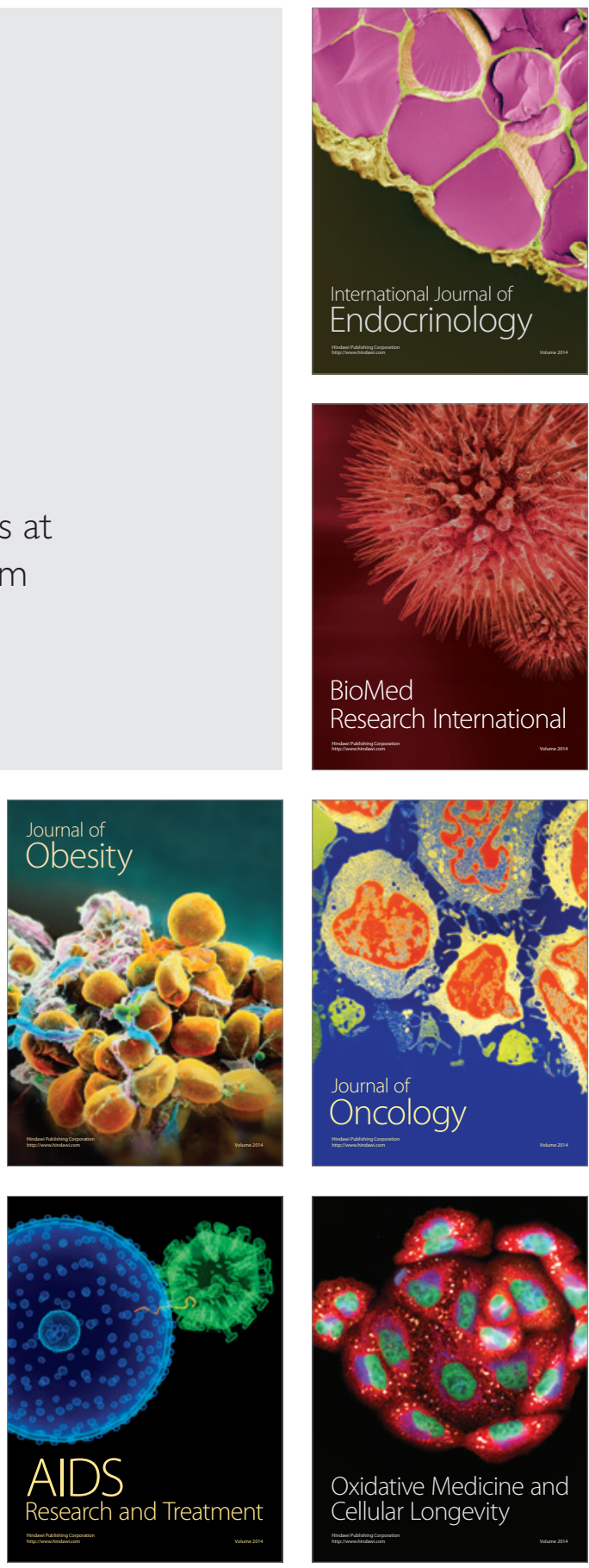